Fundación

Miguel Lillo

Tucumán

Argentina

\title{
Evidencia de puestas múltiples para la golondrina tijerita (Hirundo rustica erythrogaster, Passeriformes: Hirundinidae) en el centro de Argentina
}

\author{
Evidence of multiple clutches for the Barn Swallow (Hirundo \\ rustica erythrogaster, Passeriformes: Hirundinidae) in central \\ Argentina
}

Anabella Di Dino*1; Miguel Angel Santillán 2,4; Juan Manuel Grande 3,4

1 Facultad de Ciencias Exactas y Naturales, Universidad Nacional de La Pampa, Avenida Uruguay 151, (6300) Santa Rosa, La Pampa, Argentina.

2 Museo Provincial de Historia Natural. Secretaria de Cultura, Gobierno de La Pampa, Pellegrini 180, (6300) Santa Rosa, La Pampa, Argentina.

3 Instituto de las Ciencias de la Tierra y Ambientales de La Pampa-Consejo Nacional de Investigaciones Científicas y Técnicas de Argentina, Facultad de Ciencias Exactas y Naturales, Universidad Nacional de La Pampa, Avenida Uruguay 151, (6300) Santa Rosa, La Pampa, Argentina.

4 Colaboratorio de Biodiversidad, Ecología y Conservación, Facultad de Ciencias Exactas y Naturales, Universidad Nacional de La Pampa, Avenida Uruguay 151, (6300) Santa Rosa, La Pampa, Argentina.

* Correo electrónico: anabelladidino@gmail.com

\section{RESUMEN}

La Golodrina Tijerita (Hirundo rustica erythrogaster) es una migrante de amplia distribución en el continente americano, nidifica en el Hemisferio Norte y, desde 1980, en el Hemisferio Sur. El tamaño promedio de puesta es entre cuatro y cinco huevos. En el presente estudio reportamos puestas inusuales en nidos de la especie en el centro de Argentina, asimismo realizamos una revisión bibliográfica de los trabajos de biología reproductiva donde describan puestas de huevos de la especie y analizamos las posibles causas de dichas puestas excepcionales. El estudio se realizó durante la temporada reproductiva 2016/17, en cercanías de la ciudad de Santa Rosa, La Pampa, Argentina. Nuestros resultados muestran el primer registro de puestas de 10 y 9 huevos para América del Sur y el segundo a nivel global para la especie. Si bien no

Ref. bibliográfica: Di Dino, A., Santillán, M. A., Grande, J. M. (2021). Evidencia de puestas múltiples para la golondrina tijerita (Hirundo rustica erythrogaster, Passeriformes: Hirundinidae) en el centro de Argentina. Acta zoológica lilloana 65 (1): 33-41. doi: https://doi.org/10.30550/j.azl/2021.65.1/2021-04-06

> Recibido: 23 de diciembre 2020 - Aceptado: 6 de abril 2021.

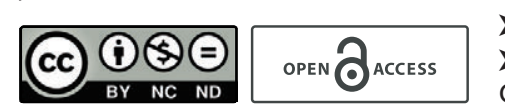

- URL de la revista: http://actazoologica.lillo.org.ar

- Esta obra está bajo una Licencia Creative Commons Atribución - No Comercial - Sin Obra Derivada 4.0 Internacional. 
se monitoreó el desarrollo de los huevos del nido, ambas indicaron que se trataban de puestas múltiples.

Palabras clave - Tamaño de puesta, cría, biología reproductiva, especie migratoria.

\section{ABSTRACT}

The Barn Swallow (Hirundo rustica erythrogaster) is a migratory bird widely distributed in the American continent, breeding in the Northern Hemisphere and, since 1980, in the Southern Hemisphere as well. The average clutch size is between four and five eggs. In the present study we report unusual clutch sizes in nests of the species in central Argentina, we also carried out a bibliographic review of studies on reproductive biology of the species where clutch sizes are reported, and we analyzed the possible causes of those exceptional clutches. The study was carried out during the reproductive season 2016/17, near the city of Santa Rosa, La Pampa, Argentina. Our results show the first record of a clutches of 10 and 9 eggs for the species in South America and the second worldwide. Although breeding on the nest was not intensively monitored both indicated that they were multiple clutches.

Keywords - Clutch size, breeding, reproductive biology, migrant species.

\section{INTRODUCCIÓN}

La golondrina común (Hirundo rustica) es un ave insectívora de hábitos migratorios, con una de las mayores áreas de distribución en el mundo (Brown y Brown, 1999; Turner, 2006; Heagy et al., 2014). Se han descrito siete subespecies pero solo una en el continente americano, Hirundo rustica erythrogaster Boddaert (1783) comúnmente llamada golondrina tijerita en Argentina (Zink, Pavlova, Rohwer, Drovetski, 2006). La especie utiliza estructuras artificiales como edificios, graneros, puentes y alcantarillas de rutas para criar (Brown y Brown, 1999; Turner, 2006; Heagy et al., 2014). El tamaño promedio de puesta es entre cuatro y cinco huevos (Brown y Brown, 1999; Heagy et al., 2014).

Tradicionalmente, solo criaba en América del Norte (entre mayo y agosto) e invernaba (septiembre-febrero) en el centro y sur de América (Turner, 2006). Pero en 1980, se encontraron seis parejas de H. r. erythrogaster nidificando en Mar Chiquita, provincia de Buenos Aires (Martínez, 1983), dentro del área histórica de distribución invernal de la especie. Desde entonces, el monitoreo de la especie ha demostrado que la población reproductora ha aumentado en número y en área de distribución, registrándose su nidificación en gran parte de la provincia de Buenos Aires (Fiameni, 2001; Morici, 2009; Billerman, Huber, Winkler, Safran, Lovette, 2011; Idoeta, Roda, Roesler, 2011; Larracoechea, Durán, D’Acunto, 2013; Grande et al., 2015; Gandoy, Delhey, Winkler, Mangini, Areta, 2019), en zonas de La Pampa (Morici, 2012; Grande et al., 2015; Bianchini, 2016, 2019), Río Negro (Segura, 2017; Petracci, León, Pérez, 2018; Bianchini, 2016, 2019), Chubut (Petracci, León, Pérez, 2019), Neuquén 
(Bianchini, 2019), Santa Fe (Juárez y Gandoy, 2019; Juárez, 2019, 2020), Córdoba (Juárez, 2019; Torres y Brandolin, 2020) y San Luis (Torres y Brandolin, 2020). Estos registros corresponden a cuatro ecorregiones: Llanura Pampeana, Espinal, Monte y Chaqueña (Burkart, Bárbaro, Sánchez, Gómez, 1999; Oyarzabal et al., 2018).

En el presente estudio, reportamos puestas inusuales en nidos de la especie en una zona de expansión poblacional en el centro de Argentina. Además, realizamos una revisión bibliográfica de los trabajos de biología reproductiva donde se describen tamaños de puestas de la especie y analizamos las posibles causas de dichas puestas y si realmente corresponden a tamaños excepcionales.

\section{MÉTODOS Y ÁREA DE ESTUDIO}

Se muestrearon las alcantarillas y puentes de ruta que son los sitios de reproducción más utilizados por la especie en Argentina (Grande et al., 2015; Gandoy et al., 2019). Se realizó en dos áreas, una hacia el oeste y suroeste de la ciudad de Santa Rosa (36 37'10.01" S, 64 $16^{\circ}$ '59.99” O), y la otra al noreste de la misma ciudad, en la provincia de La Pampa, Argentina. El sitio del oeste y suroeste abarca $85 \mathrm{~km}$ de la ruta 14 (desde el km 95 al 180) y $85 \mathrm{~km}$ de la Ruta 13 (desde el km 75 al 160), se encuentra dentro de la ecorregión del Espinal (Burkart et al., 1999), en la "unidad de bosque de esclerófitas con Prosopis caldenia" (Oyarzabal et al., 2018). El sitio del noreste comprende $56 \mathrm{~km}$ de la Ruta 7, $56 \mathrm{~km}$ de la Ruta 10 y $26 \mathrm{~km}$ de caminos vecinales, se encuentra dentro de la ecorregión de la Llanura pampeana (Burkart et al., 1999), en la "unidad de estepa psamofítica de Sorghastrum pellitum y Elionurus muticus" (Oyarzabal et al., 2018). Sin embargo, en ambas zonas de estudio, la vegetación nativa ha sido sustituida por cultivos y pasturas para la ganadería, sobre todo y casi en su totalidad, en el caso de los pastizales naturales de la Llanura pampeana (Lorda, Roberto, Bellini-Saibene, Sipowicz y Belmonte, 2008).

Las alcantarillas utilizadas para el estudio se muestrearon durante la temporada reproductiva 2016/17. Las observaciones se realizaron de manera directa o utilizando un espejo. En cada visita se registró la cantidad de nidos presentes por alcantarillas y el contenido de cada nido: cantidad de huevos o pichones. Para la revisión bibliográfica se emplearon buscadores como "Google Académico" (Google Académico, 2019), "Web of Science" (Web of Science, 2019), Searchable Ornithological Research Archive (SORA; http://elibrary.unm.edu/sora) y ResearchGate (www.researchgate.net), usando como palabras claves "Hirundo rustica", "Barn Swallow breeding biology", para poder realizar una búsqueda de los trabajos publicados sobre historia natural y cría de la especie en todo su rango de distribución.

\section{RESULTADOS Y DISCUSIÓN}

Durante la temporada reproductiva $2016 / 17$ se muestrearon 45 alcantarillas en el sitio oeste y suroeste en tres ocasiones ( 2 y 28 de diciembre de 2016 y el 29 de enero de 2017) y 55 en el sitio noreste en dos ocasiones ( 23 de diciembre de 2016 y 20 de enero de 2017). Se registraron 615 nidos, de los cuales 133 nidos contenían huevos 
y 112 contenían pichones. En dos de estos nidos se registraron puestas de inusual tamaño. En uno de los nidos, se halló el 2 de diciembre de 2016, una nidada de gran tamaño, con 10 huevos (Figura 1). La alcantarilla de hormigón armado mide $80 \mathrm{~cm}$ de alto y se localiza a $36^{\circ} 42^{\prime} 35.00^{\prime \prime} \mathrm{S}, 64^{\circ} 44^{\prime} 43.75^{\prime \prime} \mathrm{O}$, en el sitio oeste en la ecorregión del Espinal, aunque, en los alrededores de la alcantarilla, se observan cultivos y, en un radio de $1 \mathrm{~km}$ aprox., se localizan algunos parches de bosques de caldén (Prosopis caldenia). En total, había cinco nidos, dos en la cara este de la alcantarilla, ambos poseían excremento de roedor, y tres del lado oeste, uno con pluma, otro con pasto y el nido con 10 huevos. En la segunda visita (28 de diciembre de 2016), el nido de 10 huevos contenía en esa ocasión excrementos de roedor, al igual que los del lado este. Si bien, el lapso de tiempo entre las dos visitas haría factible que un número indeterminado de pichones hubieran volado del nido, la ausencia de heces de golondrina en el nido y en su zona inferior, indican que el nido no fue exitoso y que probablemente, haya sido depredado por los roedores. Así, de los cinco nidos, solo quedó uno con plumas, uno con cuatro huevos y tres con excremento de roedor.

La otra puesta inusual fue de un nido que contenía siete huevos, se registró el 23 de diciembre de 2016. La alcantarilla mide $120 \mathrm{~cm}$ de alto, ubicada a $36^{\circ} 10^{\prime} 34.01^{\prime \prime}$ S, 64 2’20.72" O en el sitio noreste en la ecorregión de la Llanura pampeana, sin embargo, en los alrededores de la alcantarilla, se visualizan cultivos. En la alcantarilla se encontraban en total ocho nidos activos y cuatro inactivos, y se visualizaron huellas de felino, posiblemente de Leopardus geoffroyi (gato montés) y de Lycalopex gymnocercus (zorro gris pampeano). En la visita posterior (20 de enero de 2017), el

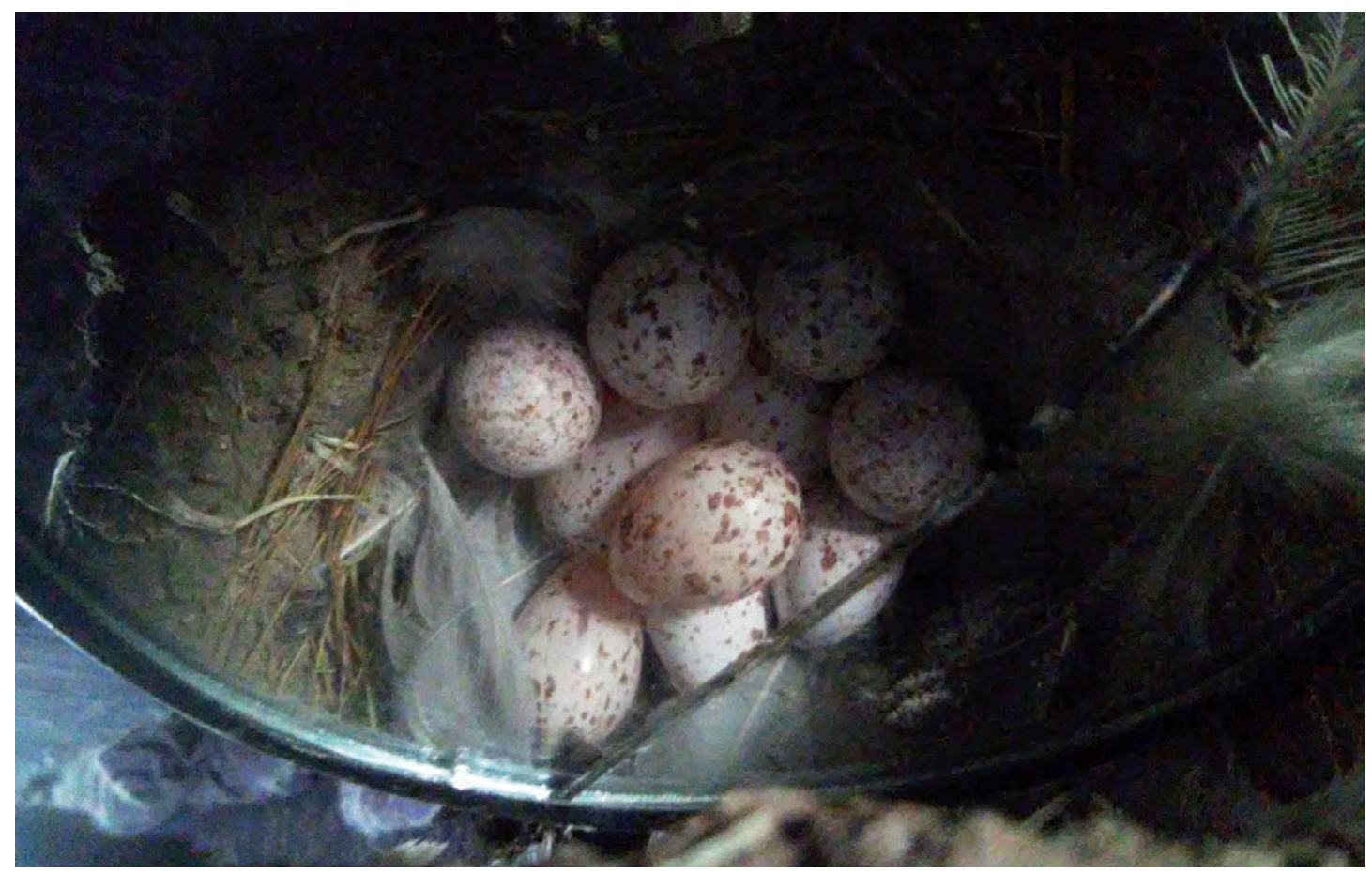

Figura 1. Nido con 10 huevos de golondrina tijerita (Hirundo rustica erythogaster), 2 de diciembre de 2016, a 41 km de Santa Rosa, La Pampa, Argentina.

Figure 1. Nest with 10 eggs from Barn Swallow (Hirundo rustica erythogaster), December 2, 2016, $41 \mathrm{~km}$. from Santa Rosa, La Pampa, Argentina. 
nido poseía nueve huevos, teniendo en cuenta el lapso de tiempo, indicaría que de los siete huevos alguno o ninguno eclosionó quedando en el nido en la segunda puesta, resultando en los nueve huevos observados. En Europa, con Hirundo rustica rustica en varias ocasiones se han observado que no se retiren los huevos de la puesta anterior quedando en el nido (Pavón y Medina, 2008), o también casos de parasitismo intraespecífico de cría, donde las hembras visitan los nidos vecinos para evaluar su idoneidad y poner sus huevos en ellos cuando la hembra del hospedador está ausente (Brown y Brown, 1999), esto ocurre regularmente en colonias mayores a cinco nidos en Dinamarca (Møller, 1987) y se ha descripto un nido con seis huevos y tres pichones que luego fracasó en Irlanda (Smiddy y O’Halloran, 2010). En cambio, para $H$. r. erythrogaster solo se ha registrado dos nidos con puestas múltiples, uno con nueve y otro con ocho huevos, en Oklahoma (Iverson, 1984) y no se han registrado parasitismo intraespecífico. De modo que el segundo nido hallado era una clara evidencia de puesta múltiple. Para el caso del nido con 10 huevos, se descarta la posibilidad que fuese parasitismo intraespecífico, debido a que se da en mayor proporción en colonias mayores a cinco nidos (Møller, 1987) y en la alcantarillas probablemente solo eran tres los nidos activos. Asimismo, otro método para determinar si la puesta pertenece a la misma hembra es observando la variación en la tonalidad de los huevos, propia de la especie (Torres y Brandolin, 2020), en ese caso, si bien en la Figura 1 no todos los huevos se visualizan completamente, los cuatro huevos superiores de la derecha poseen mayor concentración y tamaño de manchas que el huevo superior izquierdo y los cinco huevos que se encuentran debajo. Por lo tanto, se descarta la posibilidad de que fuese una puesta de gran tamaño y se confirma que también puede tratarse de una superposición de puesta como en Pavón y Medina (2008), ya sea por la misma pareja que fracasó en la puesta anterior o por otra pareja que reutilizó el nido abandonado.

A partir de la revisión bibliográfica encontramos que, hasta la fecha, para Argentina se ha descripto la presencia máxima de seis huevos en un nido (Gandoy et al. 2019). Si bien, uno de los autores de este artículo (JMG) ya había detectado un nido con siete y otro con ocho huevos en nuestra zona de estudio (datos no publicados). En Norte América, hay mayor cantidad de trabajos sobre biología reproductiva (y en general, más detallados) que, en Argentina, sin embargo, solo hay un trabajo en el que se reporte un tamaño de puesta de 10 huevos en Canadá (Campbell, Dawe, McTaggart-Cowan, Cooper, Kaiser, 1997) que probablemente haya sido, como en nuestro caso, una puesta múltiple. Y para las subespecies estudiadas en Europa y Asia, donde también el volumen de trabajos de alta calidad publicados es mayor que en Argentina, solo se registraron dos trabajos donde se mencionan dos nidos con ocho huevos, uno en Dinamarca (Møller, 2006) y otro en Polonia (Nicyñska-Bubel y Kopij, 2007).

Nuestros resultados muestran el primer registro de dos puestas múltiples de 10 huevos y 9 huevos para América del Sur y el segundo a nivel de especie. Aunque todo indicaría que las puestas no fueron exitosas. De hecho, de los 112 nidos en los que se registraron pichones volantones, el máximo número de pichones encontrados en los mismos fue de seis. Raramente, las nidadas de seis tienen un $100 \%$ de supervivencia (Mason, 1953; Brown y Brown 1999) pero se han registrado hasta siete 
pichones (Salvadori, 2009), siendo el tamaño del nido uno de los factores limitantes en la cantidad de pichones por nido (Møller, 1982).

Si bien, no se monitoreó el desarrollo de los huevos del nido, es interesante aportar este dato reproductivo a la especie en Argentina, dado que la mayoría de los trabajos evalúan variables cualitativas como, presencia/ausencia de nidos y características de los mismos (Martínez, 1983; Fiameni, 2001; Larracoechea et al., 2013; Morici, 2009; Idoeta et al., 2011; Morici, 2012; Bianchini, 2016, 2019; Segura, 2017; Petracci et al., 2018, 2019; Juárez y Gandoy, 2019; Juárez, 2019, 2020; Torres y Brandolin, 2020), de los factores que influyen en la selección del sitio de nidificación en Grande et al. (2015), de las rutas migratorias y patrones de muda (García-Pérez, Hobson, Powell, Still, Huber, 2013; Winkler et al. 2017) y un solo trabajo, hasta el momento, se ha centrado en estudiar el éxito reproductivo (Gandoy et al., 2019). Además, al ser una especie que reutiliza sus nidos, es importante tener en cuenta situaciones como las puestas múltiples, no solo documentado en este estudio, sino también en Europa (Møller, 1987; Pavón y Medina, 2008; Smiddy y O’Halloran, 2010) y América del Norte (Iverson, 1984). En este sentido recomendamos, cuando no se puede realizar un seguimiento continuo de los nidos, observar la coloración y tonalidades de los huevos para evitar errores en el análisis y estimaciones de los parámetros reproductivos, como también lo utilizó Bianchini (2017) para dos puestas de Vanellus chilensis chilensis y V. c. lampronotus (tero común). Tener presente estos datos, no solo serían datos importantes para tener en cuenta en esta especie, sino también para cualquier especie que reutilicen sus nidos.

\section{AGRADECIMIENTOS}

Agradecemos a la Facultad de Ciencias Exactas y Naturales de la Universidad de La Pampa por su apoyo institucional, a todos aquellos que ayudaron en la recolección de datos. Y especialmente a los revisores por los aportes a este trabajo.

\section{FINANCIAMIENTO}

El financiamiento fue proporcionado por la Facultad de Exactas y Naturales de la Universidad Nacional de La Pampa.

\section{PARTICIPACIÓN}

AD realizó el muestreo de campo, análisis de datos y redacción del manuscrito. MAS participó en el análisis de datos y redacción del manuscrito. JMG participó en los muestreos de campo y redacción del manuscrito.

\section{CONFLICTOS DE INTERÉS}

No existe conflicto de interés entre autores o con terceros. 


\section{LITERATURA CITADA}

Bianchini, M. (2016). Expansión del área geográfica reproductiva de la Golondrina Tijerita (Hirundo rustica erythrogaster) en las provincias de La Pampa y Río negro, Argentina. Historia Natural 6, 119-134.

Bianchini, M. (2017). Nidada atípica de tero común (Vanellus c. chilensis y V. C. lampronotus, Aves: Charadriidae) en Neuquén y Buenos Aires, Argentina. Acta Zoológica Lilloana 61: 147-156.

Bianchini, M. (2019). Novedades sobre la expansión del área geográfica reproductiva y biología básica de la Golondrina Tijerita (Hirundo rustica erythrogaster) en la Patagonia Argentina. Nótulas Faunísticas-Segunda Serie, 264, 1-10.

Billerman, S. M., Huber, G. H., Winkler, D. W., Safran, R. J., Lovette, I. J. (2011). Population genetics of a recent transcontinental colonization of South America by breeding Barn Swallows (Hirundo rustica). Auk, 128, 506-513.

Brown, C. R., Brown, M. B. (1999). Barn Swallow (Hirundo rustica). In 'The Birds of North America.' (Eds A. Poole and F. Gill.) No. 452, pp. 1-32. (The Birds of North America Inc.: Philadelphia, PA.)

Burkart, R., Bárbaro, N. O., Sánchez, R. O. Gómez, D. A. (1999). Ecorregiones de la Argentina, Buenos Aires, Administración de Parques Nacionales.

Campbell, R. W., Dawe, N. K., McTaggart-Cowan, I., Cooper, J. M., Kaiser, G. W. (1997). The Birds of British Columbia. Volume 3. Passerines: Flycatchers through Vireos. University of British Columbia Press, Vancouver. 693 pp.

Fiameni, M. (2001). Nuevos registros de nidificación de la Golondrina Tijerita ( $H i-$ rundo rustica) en la Argentina. Nuestras Aves, 42, 13.

Gandoy, F. A, Delhey, K., Winkler, D. W, Mangini G., Areta, J. I. (2019). Lower breeding success in a new range: No evidence for the enemy release hypothesis in South American Barn Swallows. The Auk, Volume 136, Issue 4. ukz050, https://doi.org/10.1093/auk/ukz050.

Grande, J. M., Santillán, M. A., Orozco, P. M., Liébana, M. S., Reyes, M. M., Galmes, M. A., Ceregheti, J. (2015). Barn Swallows keep expanding their breeding range in South America. Emu - Austral Ornithology 115, 256-260.

Heagy, A., Badzinski, D., Bradley, D., Falconer, M., McCracken, J., Reid, R.A.,Richardson, K. (2014). Recovery Strategy for the Barn Swallow (Hirundo rustica) in Ontario. Ontario Recovery Strategy Series. Prepared for the Ontario Ministry of Natural Resources and Forestry, Peterborough, Ontario. vii +64 $\mathrm{pp}$.

Idoeta, F. M., Roda, M. A., Roesler, I. (2011). La Golondrina Tijerita Hirundo rustica sigue expandiendo su área de nidificación en Argentina. Cotinga, 33, 112-114.

Iverson, S. S. (1984). Ecology of culvert-nesting Oklahoma Barn Swallows (Hirundo rustica). Thesis Doctoral, Oklahoma State University, Oklahoma City, OK, USA.

Juárez, M. (2019). Nuevos registros de nidificación de la Golondrina Tijerita (Hirundo rustica erythrogaster) en las provincias de Santa Fe y Córdoba, Argentina. Nótulas faunísticas - Segunda Serie 281, 1-5. 
Juárez, M. (2020). ¿Doble postura de la Golondrina Tijerita (Hirundo rustica) en la provincia de Santa Fe? Nuestras Aves, 65, 70.

Juárez, M., Gandoy, F. (2019). Primeros registros de nidificación de Golondrina Tijerita (Hirundo rustica) para la provincia de Santa Fe, Argentina. Nuestras Aves, 64, 10-12

Larracoechea, G., Durán, H., D’Acunto, C. (2012). Nidificación de la Golondrina Tijerita (Hirundo rustica) en el Balneario Arroyo Pareja, Buenos Aires, Argentina. Nuestras Aves, 57, 18-19.

Lorda, H., Roberto, Z., Bellini-Saibene, Y., Sipowicz, A., Belmonte, M. L. (2008). 'Descripción de las Zonas y Subzonas Agroecológicas RIAP. Área de Influencia de la EEA Anguil. (Instituto Nacional de Tecnología Agropecuaria: Anguil, La Pampa, Argentina).

Martínez, M. M. (1983). Nidificación de Hirundo rustica erythrogaster (Boddaert) en la Argentina. (Aves, Hirundinidae). Neotrópica, 29, 83-86.

Mason, E. A. (1953). Barn Swallow life history data based on banding records. BirdBanding, 24, 91-100.

Møller, A. P. (1982). Clutch size in relation to nest size in the Swallow Hirundo rustica. Ibis, 124, 339-343.

Møller, A. P. (1987). Intraspecific nest parasitism and anti-parasite behaviour in swallows, Hirundo rustica. Anim. Behavior, 35, 247-254.

Møller, A. P. (2006). Interval between clutches, fitness, and climate change. Behavioral Ecology, 18, 62-70.

Morici, A. (2009). Nidificación de la Golondrina Tijerita (Hirundo rustica) en el partido de Puán, Buenos Aires, Argentina. Nuestras Aves, 54, 35-36.

Morici, A. (2012). Primeros registros de nidificación de la Golondrina Tijerita $(H i-$ rundo rustica) en la provincia de La Pampa, Argentina. Nótulas Faunísticas, 96, $1-7$.

Ničyñska-Bubel, J., Kopij, G. (2007). Population density, nesting sites and breeding success in barn swallows in urban habitats, SW Poland. Áepkyò. 16 Bèï, 2, 232- 239

Oyarzabal, M., Clavijo, J., Oakley, L., Biganzoli, F., Tognetti, P., Barberis, I., Maturo, H.M., Aragón, R., Campanello, P.I., Prado, D., Oesterheld, M., León, R.J. (2018). Unidades de vegetación de la Argentina. Ecología Austral, 28, 040-063. https://doi.org/10.25260/EA.18.28.1.0.399.

Pavón, P., Medina, N. G. (2008). Biología reproductiva de la golondrina común ( $H i$ rundo rustica en la Universidad Autónoma de Madrid. Anuario ornitológico de Madrid, 2007-2008, 80-91.

Petracci, P. F., León, M. W., Pérez, C. H. F. (2018). La población nidificante de Golondrina Tijerita (Hirundo rustica erythrogaster) inicia la colonización de la Patagonia Argentina. Nótulas Faunísticas - Segunda Serie, 230, 1-6.

Petracci, P. F., León, M. W., Pérez, C. H. F. (2019). Primer registro de nidificación de golondrina tijerita (Hirundo rustica erythrogaster) en la provincia del Chubut, Patagonia Argentina. Nótula Faunística Segunda Serie, 262, 1-5.

Salvadori, A. (2009). A study of Barn Swallow nestings during the summer of 2008 in Ontario. North American Bird Bander, 34, 160-164. 
Segura, L. N. (2017). Southward breeding range expansion in Argentina and first breeding record of Barn Swallow Hirundo rustica in Patagonia. Cotinga, 39, 60-62.

Smiddy, P., O'Halloran, J. (2010). Breeding biology of Barn Swallows Hirundo rustica in Counties Cork and Waterford, Ireland, Bird Study, 57, 256-260. Doi: 10.1080/00063650903499594.

Torres, C., Brandolin, P. (2020). Datos sobre la biología de Hirundo rustica en el suroeste de la provincia de Córdoba. Cotinga, 42, 61-65.

Turner, A. (2006). The Barn Swallow. Poyser, London.

Winkler, D. W., Gandoy, F. A., Areta, J. I., Iliff, M. J., Rakhimberdiev, E., Kardynal, K. J., Hobson, K. A. (2017). Long-Distance Range Expansion and Rapid Adjustment of Migration in a Newly Established Population of Barn Swallows Breeding in Argentina. Current Biology, 27, 1080-1084.

Zink, R. M., Pavlova, A., Rohwer, S., Drovetski, S. V. (2006). Barn swallows before barns: population histories and intercontinental colonization. Proc. R. Soc. B., $273,1245-1251$. 